\title{
Analytical Model of Multihop IEEE 802.15.4 with Unslotted CA/CSMA
}

\author{
Ola Hussein, Nayera M. Sadek, Said Elnoubi, and Mohamed R. M. Rizk
}

\begin{abstract}
Wireless sensor networks have many applications such as industrial control, building automation, environmental monitoring, etc. IEEE 802.15.4 is a standard defined for WSN MAC layer. We present a new analytical model to evaluate the behavior of multihop IEEE 802.15.4 with unslotted CA/CSMA. Our objective is finding an expression of the busy channel probability as a function of the transmission probabilities of neighboring nodes. We join the MAC layer and traffic flow in this model and apply it to mesh network. OPNET is used as a simulation tool. The model is validated by evaluating the node throughput and the results are acceptable.
\end{abstract}

Index Terms-Analytical model, OPNET, IEEE802.15.4, wireless sensor networks.

\section{INTRODUCTION}

The IEEE 802.15.4 standard was formed for the specification of low data rate wireless sensor networks, low power and very low complexity [1]. It defines the protocols used for the physical (PHY) and medium access control (MAC) layer functionalities. The MAC layer has two types of CSMA/CA algorithms: slotted and unslotted. The IEEE 802.15.4 MAC layer supports several network topologies: star topology as single hop, and mesh and cluster tree based topologies as multihop. In general, a sensor network is multihop network with the presence of hidden nodes.

Many analytical models studied the IEEE 802.15.4 performance. A large portion of the models proposed are derived from the model proposed by Bianchi [2]. For star topology, a Markov model for IEEE 802.15.4 slotted mode in [3] studied the delay, throughput, and energy consumption with saturated throughput. The model presented in [4] considered the unsaturated and acknowledged traffic and packet retransmissions after a channel access failure for slotted CSMA/CD. The work of Di Marco et al. in [5] modeled the IEEE 802.15.4 nonbeacon enabled protocol in multihop networks. An analytical model in [6] estimated the probability of channel access failures in multihop topologies considering the probability of channel busy assessment as a function of the network traffic, and the backoff window size. An approximate analytical technique for the multihop networks based on beaconless CSMA/CA was developed in [7]. They apply this approach to the performance analysis of

Manuscript received January 10, 2014; revised March 13, 2014.

Ola Hussein is with the Alexandria Institute of Engineering and Technology, Egypt (e-mail: eng_electola@yahoo.com).

Nayera Sadek is with the Alexandria University.

Said Elnoubi is with the Electrical Engineering Department, Alexandria University.

M. R. M. Rizk is with the Virginia Polytechnic and State University, Virginia, U.S.A. tree networks rooted at a data sink.

In this work, we propose a new analytical model of multihop to study the behavior of IEEE 802.15.4 with unslotted CA/CSMA using the two dimension Markov chain. The model expresses the probability of busy channel for a single node in terms of probability of transmission of the neighboring nodes taking into account the hidden nodes. Neighboring nodes are the nodes in the carrier sensing range of this single node. We classify the neighboring node into three sets: independent set (IS), the set containing each node which is not within the carrier sensing range of each other, clique set (CS), containing each node which is within the carrier sensing range of each other, and the set of nodes which are not in independent or clique sets (not IS or CS). The analytical model joints between the traffic flow (routing path) and the MAC layer behavior. We validate the model by studying the throughput of the network nodes and comparing the analytical results with the simulation of the OPNET [8] simulation tool.

The rest of the paper is organized as follows: Section II introduces the analytical model using the two dimension Markov chain. Section III presents the derivation of the probability of busy channel for a single node and the node throughput. Section IV presents the simulated results. Section $\mathrm{V}$ concludes the paper.

\section{ANALYTICAL MODEL}

Many models describe the behavior of IEEE 802.15.4 MAC layer by a Markov chain model [3]-[7]. This paper uses the Markov chain model shown in Fig. 1. The backoff time slot is considered as the unit of the model for all probabilities and durations. Any node has three states: idle state (the node is waiting for generating a new packet to be sent), backoff state (the node accessing the channel and contenting its neighbors) or transmission state (the node is transmitting the packet). In this model, the stochastic process is represented such that,

$$
X(t)=\left\{\begin{array}{lr}
b_{i, j} & i \in\{0, \ldots m\}, j \in\left\{0, \ldots w_{i-1}\right\} \\
i d l e & k \in\left\{0, \ldots L_{s}\right\}
\end{array}\right.
$$

where,

$i \rightarrow$ backoff stage.

$j \rightarrow$ backoff counter.

$b_{i, j} \rightarrow$ backoff state.

$W i \rightarrow$ maximum duration of backoff stage and $W i=\min$ $\left\{2^{\text {BEmax }}, 2^{\text {BEmin }+i}\right\}$.

$B E_{\min } \rightarrow$ minimum number of backoffs.

$m \rightarrow$ maximum number of backoffs $\left(B E_{\max }\right)$. 
$L_{S} \rightarrow$ duration of packet transmission expressed in backoff time slot units.

$k \rightarrow$ current packet slot being transmitted.

$t_{k} \rightarrow$ transmission state.

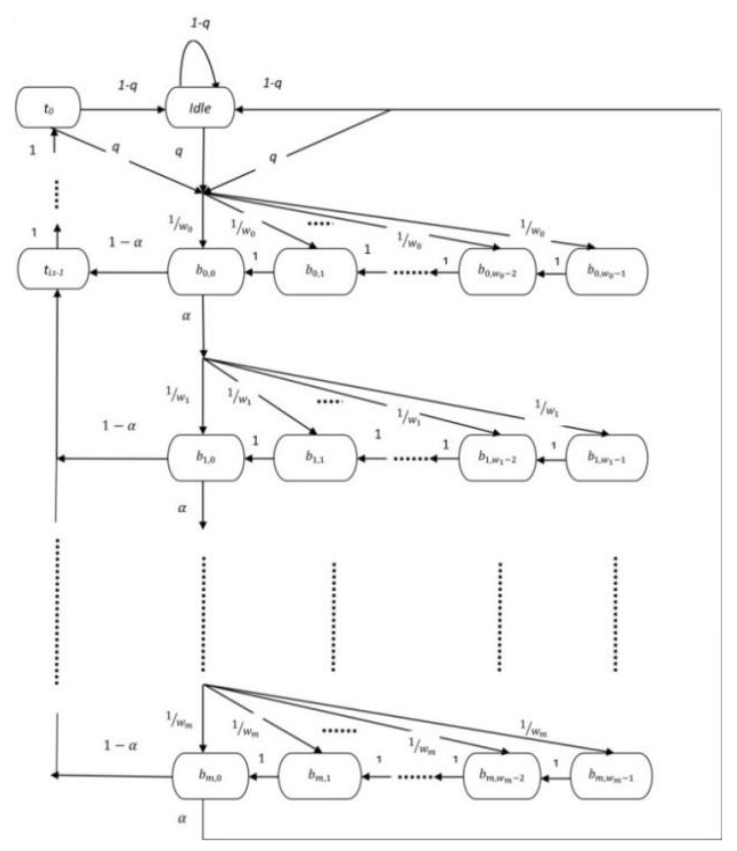

Fig. 1. Markov chain model of CSMA/CA behavior.

A closed form expression for such distribution chain is derived. Let the steady state probabilities of the Markov chain $b_{s}=p(\{X(t)=s\})$, for any state $s$. The state $s$ could be $b_{i, j}$ the backoff procedure, $b_{t_{k}}$ the packet transmission or $b_{i d l e}$ the idle state. According to the chain regularities, we have,

$$
b_{i, j}=\frac{w_{i}-j}{w_{i}} b_{i, 0} \quad 0 \leq j<w_{i}
$$

where,

$$
\begin{gathered}
w_{i}=\left\{\begin{array}{lc}
2^{i} w_{0} & i \leq B E_{\max }-B E_{\text {min }} \\
2^{\left(B E_{\max }-B E_{\min }\right)} & i \leq B E_{\max }-B E_{\min }
\end{array}\right. \\
b_{i, 0}=\alpha^{i} b_{0,0} \\
\text { for } i \leq m
\end{gathered}
$$

where $\alpha$ is the probability that the node has busy channel assessment. If $q$ is the probability of a generating a new packet in each time unit and by the normalization condition,

$$
\sum_{i=0}^{m} \sum_{j=0}^{w_{i}-1} b_{i, j}+\sum_{k=0}^{L_{s}} b_{t_{k}}+b_{i d l e}=1
$$

Equation (4) can be written in terms of $b_{0,0}$,

$$
\begin{gathered}
\sum_{i=0}^{m} \sum_{j=0}^{w_{i}-1} b_{i, j}=\sum_{i=0}^{m} \sum_{j=0}^{w_{i}-1} \frac{w_{i}-j}{w_{i}} b_{i, 0}=\sum_{i=0}^{m} \frac{w_{i}+1}{2} b_{i, 0}= \\
\sum_{i=0}^{m} \frac{w_{i}+1}{2} \alpha^{i} b_{0,0} \\
\sum_{k=0}^{L_{s}} b_{t_{k}}=L_{s} b_{t_{0}}=L_{s}\left(1-\alpha^{m+1}\right) b_{0} \\
b_{\text {idle }}=\frac{(1-q)}{q}
\end{gathered}
$$

The probability to reach an idle state depends on three different state transitions,

$$
b_{i d l e}=(1-q) b_{i d l e}+(1-q) \alpha b_{m, 0}+(1-q) b_{t_{0}}
$$

The result is,

$$
b_{0,0}=\left[\frac{1-q}{q}+L_{s}\left(1-\alpha^{m+1}\right)+\sum_{i=0}^{m} \frac{w_{i}+1}{2} \alpha^{i}\right]^{-1}
$$

Now, let $\tau$ be the probability that a node attempts or starts carrier sensing (CCA)in a randomly chosen time slot, $\tau_{\mathrm{t}}$ bethe probability that a node starts packet transmission in a randomly chosen time slot, and $T$ is denoted as the probability the node to be in a transmission state so we get,

$$
\begin{aligned}
& \tau=\sum_{i=0}^{m} b_{i, 0}=\sum_{i=0}^{m} \alpha^{i} b_{0,0}=\frac{1-\alpha^{m+1}}{1-\alpha} \\
& \tau_{t}=(1-\alpha) \tau=\left(1-\alpha^{m+1}\right) b_{0,0} \\
& T=\sum_{k=0}^{L_{s}-1} b_{t_{k}}=L_{s}(1-\alpha) \tau=L_{s} \tau_{t}
\end{aligned}
$$

\section{SYSTEM MODEL}

The network is represented by a graph $G g(V, E)$ where $V=\left\{v_{1}, \ldots, v_{N}\right\}$ is the vertices representing the nodes of the network. $E$ is the edges between vertices where an edge $E(i, j)$ represents that node $i$ and node $j$ are within their cs (carrier sensing) range of each other. Assume that the cs range and the transmission range are the same. Let, for any node $v_{i}$, the neighborhood set $G_{i}$ contains all the nodes in the cs range of node $i$. Assume a symmetrical channel, such that if $v_{j} \in G_{i}$ then $v_{i} \in G_{j}$. Any node has a set neighboring nodes $G_{i}$ and number of the subsets equal $2^{\left|G_{i}\right|}$. Some of them are called independent sets which represent simultaneous transmissions occurring from one or more nodes in cs range of any node and are not competing against each other. The other subsets are called Clique sets which indicate transmission from nodes that are in cs range of each other. This case does not occur only if they start perform CCA at the same time slot and find that the medium is not busy, so the start transmission at the same time slot and collisions may occur.

Fig. 2 shows the neighbors of node n, where $G_{n}=\{a, b, c$, $d\}$, then the possible subsets are $2^{4}=16$ subsets presented as: ( \{\}$,\{a\},\{b\},\{c\},\{d\},\{a, b\},\{a, c\},\{a, d\},\{b, c\},\{b, d\},\{c$, $d\},\{a, b, c\},\{a, b, d\},\{b, c, d\},\{a, c, d\},\{a, b, c, d\})$. Empty set is not used which means the case of no transmissions.

Independent sets are $(\{a\},\{b\},\{c\},\{d\},\{a, c\},\{a, d\},\{b$, $c\},\{c, d\})$,

Clique sets are $(\{a, b\},\{b, d\})$ and the remaining of the subsets are not IS or CS.

Channel busy assessment probability $\alpha$ can be expressed as the probability that the node detect at least one transmission in its cs range from its neighbors. Let $\beta_{n}$ be the event that a node transmits a packet, the nodes in $G_{i}$ be the neighbors of node $i$ and $S_{\text {iall }}$ be the set that has all possible subsets of the $G_{i}$ except empty group. Number of these subsets is $2^{\left|G_{i}\right|}-1=U$. Using the inclusion - exclusion principle, we get channel busy assessment probability $\alpha$ can be expressed as the probability that the node detect at least one transmission in its cs range from its neighbors. Let $\beta_{n}$ be the event that a node 
transmits a packet, the nodes in $G_{i}$ be the neighbors of node $i$ and $S_{\text {iall }}$ be the set that has all possible subsets of the $G_{i}$ except empty group. Number of these subsets is $2^{\left|G_{i}\right|}-1=U$. Using the inclusion - exclusion principle, we get,

$$
\alpha_{i}=p\left\{\bigcup_{n \in G_{i}} \beta_{n}\right\}=\sum_{k=1}^{\left|G_{i}\right|}(-1)^{k-1} \sum_{\substack{\left|S_{u}\right|=k \\ S_{u} \in S_{\text {iall }}}} p\left(\bigcap_{n \in S_{u}} \beta_{n}\right)
$$

The sum runs over all subsets $S_{u}$ in $S_{\text {iall }}$ contain exactly $k$ nodes and each of these nodes has position $n \in G_{i}$. In the cases of independent set and clique sets, we use (14) and (15) respectively,

$$
\begin{gathered}
p\left(\bigcap_{n \in S_{u}} \beta_{n}\right)=\prod_{n \in S_{u}} T_{n} \\
p\left(\bigcap_{n \in S_{u}} \beta_{n}\right)=L_{s} \prod_{n \in S_{u}} \tau_{t_{n}}
\end{gathered}
$$

The remaining of the subsets depends on the network topology. Here an example according to Fig. 2 for the IS subset $=\{a, c\}$, the probability of this event is $T_{a} T_{c}$ while for the CS subset $=\{a, b\}$, the probability of this event is $L_{s} \tau_{\mathrm{t}_{\mathrm{a}}} \tau_{\mathrm{t}_{\mathrm{b}}}$ If the subset is not IS or CS as the subset $\{a, b, c\}$, then the probability of this event is $L_{s} \tau_{\mathrm{t}_{\mathrm{a}}} \tau_{\mathrm{t}_{\mathrm{b}}} \times L_{s} \tau_{\mathrm{t}_{\mathrm{c}}}$ where nodes $a$ and $b$ are within cs range of each other, so they should start transmission at the same time independently of the transmission of node $c$.

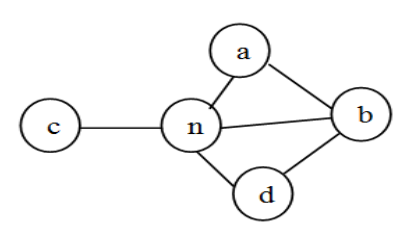

Fig. 2. Neighbors of node $n$.

Another example the subset $\{a, b, c, d\}$ which is not IS or CS. This event has the probability $L_{s} \tau_{\mathrm{t}_{\mathrm{a}}} \tau_{\mathrm{t}_{\mathrm{b}}} \tau_{\mathrm{t}_{\mathrm{d}}} \times L_{s} \tau_{\mathrm{t}_{\mathrm{c}}}$. Although node $a$ and $d$ are independent but they related to the transmission of node $b$. This group of the three nodes cannot make a transmission only if they start transmission at the same time and independently of node $c$ packet transmission.

Suppose that the total arrival rate $Q=q$ / aUnitBackoffPeriod where aUnitBackoffPeriod is the duration of the basic time unit in IEEE 802.15.4. Suppose that $\lambda$ is the packet generation rate at any node. According to the concept of the traffic flow balance, the total arrival rate at node $i$ is the sum of packet generation rate of the node itself and the aggregated traffics that are forwarded by the other nodes in the neighborhood of it. Traffic flow balance equations can be expressed in matrix notation. Suppose that $Q$ is a vector of the total arrival rate $Q=\left[Q_{1}, \ldots, Q_{N}\right]$ and the packet generation rate $\lambda=\left[\lambda_{1}, \ldots, \lambda_{\mathrm{N}}\right]$. Define a routing matrix $M \in R^{N \times N}$ where

$$
M_{i j}=\left\{\begin{array}{rr}
1 & \text { if node } j \text { destination to node } i \\
0 & \text { otherwise }
\end{array}\right.
$$

Scaling $M_{i j}$ by $R_{i j}$ yields to $f_{i j}=R_{i j} M_{i j}$, where $F$ is the flow traffic matrix. The traffic balance equations are,

$$
\begin{gathered}
Q=\lambda+Q F \\
Q=\lambda(I-F)^{-1}
\end{gathered}
$$

where $I \in R^{N \times N}$ is the identity matrix.

$R_{i k}$ is the reliability of packet transmission from node $k$ to node $i$,

$$
\begin{gathered}
R_{i j}=1-P_{c f_{i j}}-P_{a f_{i}} \\
P_{a f_{i}}=\alpha_{i}^{m+1} \\
P_{c f_{i j}}=\left(1-\alpha_{i}^{m+1}\right) P_{c o l l} i j
\end{gathered}
$$

where $P_{c f_{i j}}$ is the probability that the channel is accessed successfully but the packet transmission from node $i$ to node $j$ has a collision while $P_{a f}$ is the probability the packet is discarded due to successive CCA failures. $P_{\text {coll }}$ ij is defined as the probability of a packet collision when this packet transmits from node $i$ to node $j$. Collisions have two cases. The first case occurs when one or more nodes in the shared region between cs ranges of nodes $i$ and $j\left(G_{i} \cap G_{j}\right)$ start transmit their packets at the same time of node $i$ transmission started. The second case occurs because of the hidden nodes with respect to node $i$ that are in the cs range of node $j$, and can be defined as $\left(G_{j}-\left(G_{i} \cap G_{j}\right)\right)$. These hidden nodes engage the medium with a packet transmission to be busy when node $i$ makes a transmission to node $j$. Let $P_{h x}$ the probability of collision due to hidden nodes and $P_{s x}$ the probability of collision due to shared nodes.

$$
\begin{gathered}
P_{\text {coll }_{i j}}=P\left(P_{h x_{i j}} \cup P_{s x_{i j}}\right)=P_{h x_{i j}}+P_{s x_{i j}}-P_{h x_{i j}} P_{s x_{i j}} \\
P_{s x_{i j}}=\tau_{t_{i}} \times\left(1-\prod_{k \in\left\{G_{i} \cap G_{j}\right\}}\left(1-\tau_{t_{k}}\right)\right) \\
P_{h x i j}=T_{i} \times P
\end{gathered}
$$

(probability that meduim busy due to hidden nodes)

$$
P_{h x_{i j}}=T_{i} \times \sum_{k=1}^{\left|G_{h i j}\right|}(-1)^{k-1} \sum_{\substack{\left|S_{u}\right|=k \\ S_{u} \in S_{i j a l l}}} p\left(\bigcap_{n \in S_{u}} \beta_{n}\right)
$$

where $G_{h i j}=G_{j}-\left(G_{i} \cap G_{j}\right)$ is the set of hidden nodes of node $i$ from neighbors of $j$ node and $S_{i j a l l}$ contain all possible subsets of $G_{h i j}$ except the empty group. Finally, the throughput of node $i$ is defined as the total traffic received successfully that routed or forwarded from the other nodes

$$
\text { Thput }_{i}=Q_{i}-\lambda_{i}
$$

To obtain the solution of the multihop network model, we solve (11), (13), (18) and (19) numerically using the built in function $f$ solve of MATLAB software.

\section{NUMERICAL AND SiMULATED RESULTS}

The model proposed is implemented using the MATLAB software. To validate our model, the results are compared with 
the results obtained from the OPNET [8].

The network used for evaluation consists of eight wireless sensor nodes with mesh network topology as shown in Fig. 3 a. The lines indicate the neighbors of each node. The carrier sensing range is $10 \mathrm{~m}$. The simulation area is $25 \mathrm{~m} \times 25 \mathrm{~m}$, and the simulation time is 500s. The IEEE802.15.4 parameters are as follows, the aUnitBackoffPeriod $=320 \mu \mathrm{s}, N B_{\max }=4$, $B E_{\max }=5, B E_{\min }=3$. No acknowledgments or retransmissions are used.

Fig. 4 shows the throughput of each node in the network at three different values of arrival rate, $\lambda=10,1,0.1 \mathrm{pkt} / \mathrm{s}$ and fixed packet length, $L_{s}=7$. The traffic flow distribution is indicated by arrows as shown in Fig. $3 \mathrm{~b}$ for $\lambda=10$ and $1 \mathrm{pkt} / \mathrm{s}$ and Fig. $3 \mathrm{c}$ for $\lambda=0.1 \mathrm{pkt} / \mathrm{s}$. The destination node is node 6 .

Fig. 4 shows that the results of both the analytical model and the simulated results from the OPNET are very close especially at the low arrival rate. It also shows that node 6 as the sink has the highest throughput while the nodes that do not forward the traffic have zero throughput.

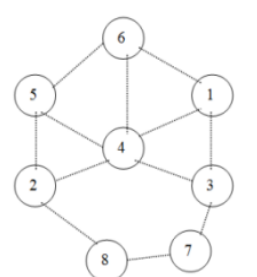

(a)

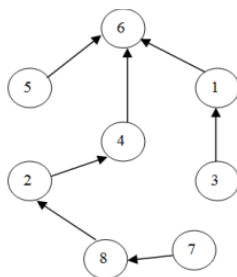

(b)

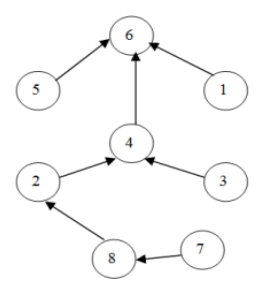

(c)
Fig. 3. (a) Main network (b) $\lambda=10,1 \mathrm{pkt} / \mathrm{s}$ (c) $\lambda=0.1 \mathrm{pkt} / \mathrm{s}$

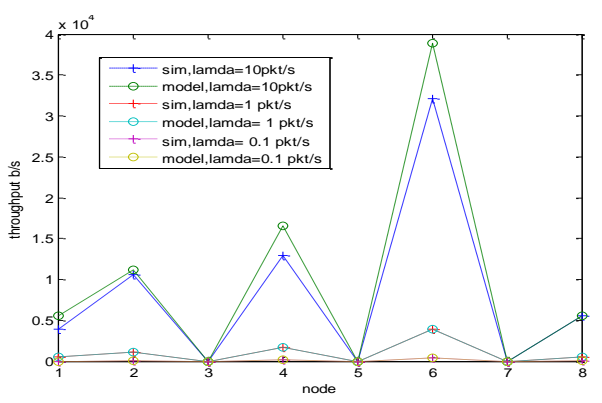

Fig. 4. Throughput of each node in the network.

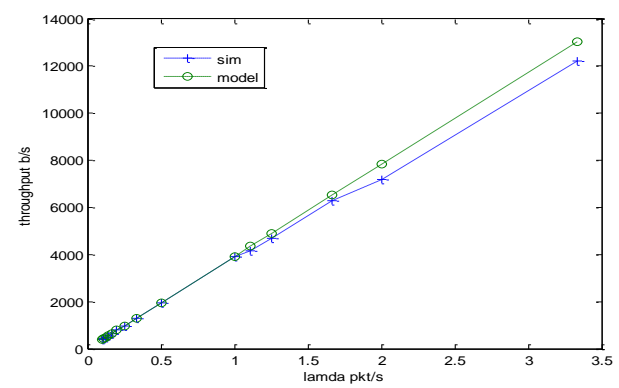

Fig. 5. Throughput of node 6 for different $\lambda$.

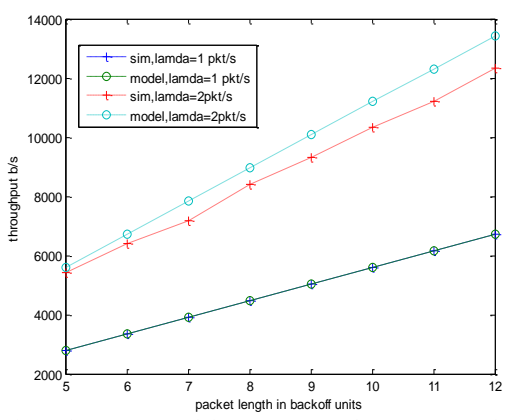

Fig. 6. Node 6 througput with $L_{s}=5$ to 12 in aUnitBackoffPeriod.
Fig. 5 shows the throughput of node 6 at different arrival rates and $L_{s}=7$. It also proves that the analytical and simulated results are very close. As the arrival rates increases, the throughput computed from our model and obtained from the OPNET increases.

Fig. 6 shows the througput of node 6 at arrival rate 1 and 2 $\mathrm{pkt} / \mathrm{s}$ and different $L_{s}$ in the range of 5 to 12 . The results indicate that the throughput of the analytical model are almost equal to the simulated one. As the packet size increases, the througput increases.

\section{CONCLUSIONS AND Future WORK}

In this paper, we propose an analytical model for the IEEE802.15.4 MAC layer using unslotted CA/CSMA. The model studies the mesh network as a multihop network and takes into account the presense of hidden terminal. It joints between the MAC and the routing paths. The analytical model is evaluated by comparing its throughput results to a simulated results obtained using the OPNET. The comparison shows that the analytical results have a good agreement with the simulated resullts. It also proves the validation of the proposed analytical model to model the IEEE 802.15.4 multihop wireless sensor networks.

Futureworks include an extensive study of another metrics and MAC performance indicators such as delay and power consumption. The model can also be modified easily to be more realistic by considering alossy channel instead of the assumption of a perfect channel.The model can also be implemented in many applications such as investigating the optimal routes for IEEE 802.15.4 multi-hop networks.

\section{REFERENCES}

[1] IEEE Std 802.15.4-2996. (September 2006). "Part 15.4: wireless medium access control (MAC) and physical layer (PHY specifications for low-rate wireless personal area networks (WPANs)." [Online]. Available: http://www.ieee802.org/15

[2] G. Bianchi, "Performance analysis of the IEEE 802.11 distributed cordination function," IEEE Journal on Selected Areas in Communications, vol. 8, issue 3, pp. 535-547, March 2000.

[3] S. Pollin, M. Ergen, S. C. Ergen, B. Bougard, L. V. D. Perre, F Catthoor, I. Moerman, A. Bahai, and P. Varaiya, "Performance analysis of slotted carrier sense IEEE 802.15.4 medium access layer," in Proc. IEEE GLOBECOM, 2006, pp. 1-6.

[4] P. Park, P. D. Marco, P. Soldati, C. Fischione, and K. H. Johansson. “A generalized Markov chain model for effective analysis of slotted IEEE 802.15.4," in Proc. the 6th IEEE Int. Conf. on Mobile Adhoc and Sensor Systems (MASS'09), 2009, pp. 130-139.

[5] P. D. Marco, P. Park, C. Fischione, and K. Johansson, "Analytical modeling of IEEE 802.15.4 for multi-hop networks with heterogeneous traffic and hidden terminals," in Proc. the IEEE Global Telecomm. Conf. (GLOBECOM 2010), 2010, pp. 1-6.

[6] E. Feo and G. A. D. Caro. (May 2011). "An analytical model for IEEE 802.15.4 non-beacon enabled CSMA/CA in multihop wireless sensor networks." Technical Report IDSIA-06-11. [Online]. Available: http://www.idsia.ch/ gianni/Papers/IDSIA-05-11.pdf

[7] R. Srivastava and A. Kumar, "Performance analysis of beacon-less IEEE 802.15.4 multihop networks," in Proc. 4th International Conference on Communication Systems and Networks (COMSNETS), IEEE, Bangalore, India, 2012, pp. 1-10.

[8] Riverbed application and network performance management solutions. [Online]. Available: http://www.opnet.com

Ola Hussein was born in Alexandria, Egypt, in November 1981. She received the B.S. and M.S. degrees from Alexandria University, Alexandria, Egypt, in 2003 and 2008, respectively. From November 2003, she is a teaching assistant at Alexandria Institute of Engineering and Technology. Her research interests include sensor networks, ad hoc networks and wireless communication. 
Nayera Sadek was born in Alexandria, Egypt, in January 1973. She received the B.S. and M.S. degrees from Alexandria University, Alexandria, Egypt, in 1995 and 1997, respectively, and the Ph.D. degree from Southern Methodist University, Dallas, TX, in 2004, all in electrical engineering. From August 2004, she is a lecturer at Alexandria University. Her research interests include sensor networks, network security, encryption and embedded systems.

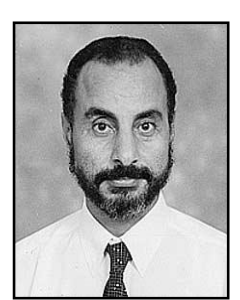

Said Elnoubi was born in Alexandria, Egypt, in September 1951. He received the B.S. and M.S degrees from Alexandria University, Alexandria, Egypt, in 1974 and 1977, respectively, and the Ph.D. degree from Southern Methodist University, Dallas, TX, in 1980, all in electrical engineering. From October 1974 to December 1977, he was an instructor at Alexandria University. From January 1978 to December 1980, he was a research assistant at Southern Methodist University. From January to August 1981, he was a postdoctoral fellow at the same university. From September 1981 to August 1987, he was an assistant professor of electrical engineering at the University of Illinois, Chicago, IL. From September 1987 to June 1989, he was an associate professor in the Electrical Engineering Department, Alexandria University. From August 1989 to May 1990, he was a visiting professor at Wichita State University,Wichita, KS. From 1990 to 1994, he was a member of the Technical Staff at MITRE Corporation, McLean, VA. Currently, he is a professor in the Electrical Engineering Department, Alexandria University. His research interests include digital modulation, mobile radio and wireless communications, spread spectrum, and air/ground radio communications. Dr. Elnoubi has been a technical reviewer of the IEEE Transactions on Communications, Aerospace and Electronic Systems, Vehicular Technology, Information Theory, and Wireless Communications, as well as the Institute of Electrical Engineers proceedings and Electronic Letters. He was an assistant editor of Communications and IEEE Transactions on Vehicular Technology. He received the Egypt National Incentive Award for Engineering Science and the Salah Amer Incentive Award for Electronic Science in 1989. He is listed in the Marquis Who's Who in the World.

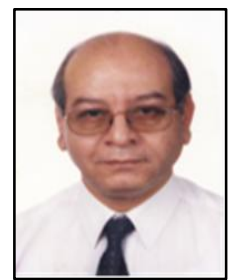

M. R. M. Rizk obtained his B.Sc. from Alexandria University and his master's and Ph.D. from McMaster University, Canada. He worked as an assistant professor at McMaster University. He was a visiting professor at Sultan Qaboos University, Oman, Beirut Arab University and the Arab Academy for Science and Technology, Egypt. He is an adjunct professor to Virginia Polytechnic and State University, Virginia, U.S.A. His research interests include computer aided design, wireless networks, encryption, fuzzy logic, and image processing. 